1919.] CORRECTIONS TO CAMBRIDGE COLLOQUIUM.

Kummer* has shown that

$$
\frac{B_{a}}{a} \equiv(-1)^{k \mu} \frac{B_{a+k \mu}}{a+k \mu} \quad(\bmod l),
$$

where $k$ is an integer and $a$ is not a multiple of $\mu=(l-1) / 2$. This gives

$$
(-1)^{s \mu} \frac{B_{(s l+1) / 2}}{s(l+1) / 2} \equiv \frac{B_{(s+1) / 2}}{(s+1) / 2}(\bmod l),
$$

and applying the latter relation to (8) for $a=1$, we obtain Kummer's result to the effect that the necessary and sufficient condition that $h$ be divisible by $l$ is that one of the Bernoulli numbers $B_{s},\left(s=1,2, \cdots, \frac{1}{2}(l-3)\right)$ is divisible by $l$.

Bala, Pa.,

November, 1918.

\title{
CORRECTIONS AND NOTE TO THE CAMBRIDGE COLLOQUIUM OF SEPTEMBER, 1916.
}

BY PROFESSOR G. C. EVANS.

1. Corrections. On page 35 in equation $\left(9^{\prime}\right)$ change $\gamma$ to $\alpha$. In all the formulas and equations following on page 35 change $\gamma$ to $\beta$ and $\beta$ to $\alpha$.

On page 37 in equation (12) change $\gamma$ to $\beta$ and $\beta$ to $\alpha$.

On page 39 in the second equation there should be an $i$ as a factor of each of the last two terms of the integrand.

2. Note to Art. 27, the Analogue of Green's Theorem. The approach to the analogue of Green's theorem is clearer if made in the following way, and bears more relation to the development with which we are familiar in calculus. The meaning of equations (17) to (20) is perhaps not clear, as the equations stand. But the invariant $H_{\Phi_{1} \Phi_{1}}$, defined by

$$
\left(V_{1} \times V_{2}\right) H_{\Phi_{1} \phi^{\prime}}=W_{1} \times W_{2}{ }^{\prime},
$$

which may be rewritten in the new forms

$$
\begin{aligned}
H_{\Phi_{1} \Phi_{1}} & =\left(\beta \cdot W_{1}\right)\left(\beta \cdot W_{1}^{\prime}\right)+\left(\alpha \cdot W_{1}\right)\left(\alpha \cdot W_{1}^{\prime}\right) \\
& =-\left(\beta \cdot W_{1}\right)\left(\alpha \cdot W_{2}^{\prime}\right)+\left(\alpha \cdot W_{1}\right)\left(\beta \cdot W_{2}^{\prime}\right),
\end{aligned}
$$

\footnotetext{
* L. c., vol. 41, p. 368.
} 
as we see below, bears a noticeable resemblance to the scalar product of two gradients which is subjected to the familiar analysis of Green's theorem.

The forms just written, however, do not lend themselves to the customary integration by parts. Let us then in these formulas replace the part which refers to, say, $W_{1}$ and $W_{2}$ by functions $\varphi_{1}$ and $\varphi_{2}$ which depend on them (retaining meanwhile the quantities $W_{1}^{\prime}$ and $\left.W_{2}^{\prime}\right)$ and seek to write $H_{\Phi_{1} \Phi_{1}}$ in the form

$$
H_{\Phi_{1} \Phi_{1^{\prime}}}=W_{1}^{\prime} \cdot \nabla \varphi_{1}+W_{2}^{\prime} \cdot \nabla \varphi_{2}
$$

or more generally, introducing an arbitrary point function $k(x, y, z)$, in the form

$$
k H_{\Phi_{1} \Phi_{1}^{\prime}}=W_{1}^{\prime} \cdot \nabla \varphi_{1}+W_{2}^{\prime} \cdot \nabla \varphi_{2} .
$$

Green's theorem is merely the result of integrating both sides of (22) over a three-dimensional region, and reducing the righthand member by an integration by parts.

What conditions must $\varphi_{1}, \varphi_{2}$ satisfy to make possible the equation (22)? A short analysis shows that a necessary and sufficient condition is given by equation (17), which may be deduced directly, starting from this point of view.

The analysis follows. In the first place, by making use of the identities (see (8) page 34)

$$
W_{1}=V_{1} \alpha \cdot W_{1}+V_{2} \beta \cdot W_{1}, \quad W_{2}^{\prime}=V_{1} \alpha \cdot W_{2}^{\prime}+V_{2} \beta \cdot W_{2}^{\prime},
$$

we have

$$
W_{1} \times W_{2}^{\prime}=\left(V_{1} \times V_{2}\right)\left\{\left(\alpha \cdot W_{1}\right)\left(\beta \cdot W_{2}{ }^{\prime}\right)-\left(\beta \cdot W_{1}\right)\left(\alpha \cdot W_{2}^{\prime}\right)\right\},
$$

whence, from the definition (21),

$$
H_{\Phi_{1} \Phi_{1}{ }^{\prime}}=-\left(\beta \cdot W_{1}\right)\left(\alpha \cdot W_{2}^{\prime}\right)+\left(\alpha \cdot W_{1}\right)\left(\beta \cdot W_{2}^{\prime}\right) .
$$

By the conditions of isogeneity $\left(10^{\prime \prime}\right)$, we get the other new form mentioned for $H_{\Phi_{1} \Phi_{1}}$, as well as additional ones of the same character.

The equation just written, by comparison with (22), gives us the equation

$$
k\left[\left(\alpha \cdot W_{1}\right)\left(\beta \cdot W_{2}^{\prime}\right)-\left(\beta \cdot W_{1}\right)\left(\alpha \cdot W_{2}{ }^{\prime}\right)\right]=W_{1}{ }^{\prime} \cdot \nabla \varphi_{1}+W_{2}{ }^{\prime} \cdot \nabla \varphi_{2} .
$$

In the right-hand member the following substitutions may be 
made, to make the two members similar in construction:

$$
\begin{array}{r}
W_{1}^{\prime} \cdot \nabla \varphi_{1}=\nabla \varphi_{1} \cdot\left(V_{1} \beta-V_{2} \alpha\right) \cdot W_{2}^{\prime} \\
\quad=\left(V_{1} \cdot \nabla \varphi_{1}\right)\left(\beta \cdot W_{2}^{\prime}\right)-\left(V_{2} \cdot \nabla \varphi_{1}\right)\left(\alpha \cdot W_{2}^{\prime}\right) \\
W_{2}^{\prime} \cdot \nabla \varphi_{2}=\nabla \varphi_{2} \cdot\left(V_{1} \alpha+V_{2} \beta\right) \cdot W_{2}^{\prime} \\
=\left(V_{1} \cdot \nabla \varphi_{2}\right)\left(\alpha \cdot W_{2}^{\prime}\right)+\left(V_{2} \cdot \nabla \varphi_{2}\right)\left(\beta \cdot W_{2}^{\prime}\right) .
\end{array}
$$

Hence the equation becomes

$$
\begin{aligned}
& \left(\nabla \varphi_{1} \cdot V_{1}+\nabla \varphi_{2} \cdot V_{2}-k \alpha \cdot W_{1}\right)\left(\beta \cdot W_{2}{ }^{\prime}\right) \\
& +\left(\nabla \varphi_{2} \cdot V_{1}-\nabla \varphi_{1} \cdot V_{2}+k \beta \cdot W_{1}\right)\left(\alpha \cdot W_{2}^{\prime}\right)=0 .
\end{aligned}
$$

By hypothesis, $\varphi_{1}, \varphi_{2}, k$ are independent of $W_{1}{ }^{\prime}, W_{2}{ }^{\prime}$; hence by putting $W_{2}^{\prime}=V_{2}$ and $V_{1}$ successively the following equations are deduced

$$
\begin{aligned}
& k \alpha \cdot W_{1}=\nabla \varphi_{1} \cdot V_{1}+\nabla \varphi_{2} \cdot V_{2}, \\
& k \beta \cdot W_{1}=\nabla \varphi_{1} \cdot V_{2}-\nabla \varphi_{2} \cdot V_{1},
\end{aligned}
$$

which with the identity $W_{1}=V_{1} \alpha \cdot W_{1}+V_{2} \beta \cdot W_{1}$ yield the result

$$
k W_{1}=\left(V_{1} V_{1}+V_{2} V_{2}\right) \cdot \nabla \varphi_{1}+\left(V_{1} V_{2}-V_{2} V_{1}\right) \cdot \nabla \varphi_{2},
$$

which is merely (17). Vice versa, from (17) may be deduced (22). Equation (18) is equivalent to (17) by the relation of isogeneity.

In a similar manner equation (19) or (20) is seen to be a necessary and sufficient condition for the functions $\varphi_{1}^{\prime}$ and $\varphi_{2}{ }^{\prime}$ to satisfy in order that $H_{\Phi_{1} \Phi_{1}}$ may be written in the form $\left(22^{\prime}\right)$.

Equation (17) is equivalent to two independent differential equations on the two functions $\varphi_{1}, \varphi_{2}$ to be determined, as is seen in the equivalence of that equation to the two preceding (i. e., the conditions in the plane $V_{1} V_{2}$ ). Hence the function $k$ remains entirely arbitrary, and may if we choose be taken everywhere positive.

Parts, France,

February, 1919. 